\title{
Anabases
}

ANABASES Traditions et réceptions de l'Antiquité

12 | 2010

Varia

\section{Charles Huber und Julius Euting in Arabien nach französischen, auch heute noch nicht veröffentlichten Dokumenten}

Hélène Lozachmeur et Françoise Briquel-Chatonnet

\section{(2) OpenEdition}

Journals

Édition électronique

URL : http://journals.openedition.org/anabases/1230

DOI : $10.4000 /$ anabases. 1230

ISSN : 2256-9421

Éditeur

E.R.A.S.M.E.

Édition imprimée

Date de publication : 1 octobre 2010

Pagination : 195-200

ISSN : $1774-4296$

Référence électronique

Hélène Lozachmeur und Françoise Briquel-Chatonnet, « Charles Huber und Julius Euting in Arabien nach französischen, auch heute noch nicht veröffentlichten Dokumenten », Anabases [Online], 12 | 2010, Online erschienen am: 01 Oktober 2013, besucht am 20 Oktober 2019. URL : http:// journals.openedition.org/anabases/1230 ; DOI : 10.4000/anabases.1230

Ce document a été généré automatiquement le 20 octobre 2019

(c) Anabases 


\title{
Charles Huber und Julius Euting in Arabien ${ }^{1}$ nach französischen, auch heute noch nicht veröffentlichten Dokumenten $^{2}$
}

\author{
Hélène Lozachmeur et Françoise Briquel-Chatonnet
}

1 Wie kann man von nordarabischer Entdeckung in Arabien während der zweiten Hälfte des XIX. Jahrhunderts reden, ohne die zwei Reisen von Charles Huber, dem Franzosen, und die Reise von Julius Euting, dem Deutschen zu erwähnen? Diese Reisen, in der Tat, stellen einen wichtigen Wendepunkt in der Prospektion von Arabien dar.

2 Zunächst möchten wir aber Miss Helen Pearson zu ihrer Dissertation „Relations between Julius Euting and Charles Huber in Arabia“ (PhD. University of Manchester, Dept. of Middle Eastern Studies, 1993) gratulieren, wenn sie auch darin eher auf die deutschen Archive zurückgreift.

3 Aber jetzt möchten wir zu unserem heutigen Thema kommen. Wir müssen in der Tat, als Historiker, diese abenteuerlichen Reisen in den Kontext des damaligen europäischen Wettbewerbs zurückversetzen. Das scheint uns hier wesentlich zu sein für die Zeit die zwischen 1870 und 1914 liegt - da Charles Huber ein hundertprozentiger patriotischer Elsässer war und das ist wahrscheinlich auch der Schlüssel zum besseren Verständnis der Verhältnisse zwischen Julius Euting und Charles Huber.

\section{Die damals existierende Lage}

4 Kurz geschildert. Einerseits: Julius Euting, ein Württemberger und wie er selber schrieb, ab 1871 „Bibliothécaire-Chef“ an der Straßburger Universitätsbibliothek, „docteur en philosophie“ und „Professeur“. Ein Gelehrter. 
5 Andererseits: Charles Huber, ein geborener Strassburger, ein Erdkundler. Er hatte sehr unter der politischen Lage, die nach dem 1870iger Krieg in Straßburg herrschte, zu leiden.

6 Er selber schrieb aus Straßburg am 14. November 1882 an Herrn Maunoir, Secrétaire général de la Société de Géographie, in Paris3: „[...] Wenn, abgesehen davon, meine Anwesenheit in Paris Ihnen erleichtern könnte, was Sie für mich tun möchten, dann lassen Sie es mich wissen, ich werde dann sobald wie möglich zu Ihnen fahren, obgleich meine Lage hier immer schlimmer wird und ich nur noch unter großen Schwierigkeiten Aufenthalts- und Abwesenheitsgenehmigungen erhalten kann ${ }^{4}$."

7 Weiter, in einem anderen Brief aus Straßburg, vom 25. November 1882, immer noch an Herrn Maunoir gerichtet, erklärt er, daß seine zwei jüngeren Brüder, die ihren Militärdienst in Frankreich absolviert hatten und dann als „réfractaires“ ins Gefängnis geworfen und mit einer Geldstrafe behaftet wurden.

8 Er fährt fort: „[...] was mich selbst betrifft, bin ich seit 1878 als Mitglied der Ligue d'Alsace sowie der Rest meiner Familie bei den Behörden schlecht angeschrieben. Bei meiner Rückkehr aus Arabien erhielt ich nur mit großer Mühe und trotz der versöhnlichen Gesinnung des Gouverneurs von Elsaß-Lothringen die Genehmigung, mich zuhause bei meiner Mutter in Straßburg aufzuhalten. [...]Jeden Montag muß ich mich bei der Polizeiprefektur melden; für jede Abwesenheit und nach jeder Rückkehr in die Stadt muß ich Erklärungen abgeben und Genehmigungen einholen. Bei meiner letzten Heimkehr aus Paris musste ich mich einem regelrechten Verhör unterziehen, worin man erfahren wollte was ich dort gemacht habe. [...] Es wurde mir nahegelegt, mich still zu verhalten, wenn ich doch noch hier bleiben will und noch einmal nach Arabien reisen will ${ }^{5}$."

9 Auch ist sein Familienstammgut, wie er selber erwähnt, seit sieben Jahren unter Zwangsverwaltung gestellt.

10 Also, zu dieser bestimmten Zeit, keine richtige Freiheit und wenige Mittel stehen diesem unternehmenden Elsässer zur Verfügung.

\section{Die erste Reise von Charles Huber}

11 Die erste Reise von Charles Huber in Arabien verlief von 1878 bis 1882 und war, in einem Wort zusammengefaßt, ein grosses und langes Unternehmen mit stark beschränkten Mitteln. Die Finanzen, die einem Forschungsreisenden zur Verfügung gestellt wurden, waren äußerst knapp und dies hat sich, nebenbei gesagt, auch bis heute noch nicht sehr verändert.

12 Der Bericht über diese Reise wurde von der Société de Géographie nur zum Teil veröffentlicht ${ }^{6}$. Charles Huber erhielt für seine Funde die Goldmedaille der großen Forschungsreisenden, die er leider durch seine Ermordung während der zweiten Reise nie persönlich entgegennehmen konnte ${ }^{7}$.

13 Nach seiner Rückkehr in Straßburg kommen in seinen Briefen im Jahr 1882 viele Sorgen zum Ausdruck, aber auch die Leidenschaft die ihn belebt.

14 Ein kurzes Beispiel aus seinem Brief vom 28. März $1882^{8}$, wo er schreibt: „[...] hier ist was ich noch zum Schluß meines Berichtes hätte hinzufügen wollen: persönlich bin ich mit dem Ergebnis meiner Mission nicht zufrieden, besonders wenn ich berücksichtige 
was noch zu tun ist und was ich sicher bin, in Arabien noch tun zu können, und was ich getan hätte wenn man mir nochmals einen kleinen Zuschuß gewährt hätte. Ihnen, Monsieur, brauche ich nicht zu beweisen welchen Nutzen es bringen würde, meine erste Reiseroute wieder aufzunehmen und fortzusetzen, ab A'neïzah' über Ryadh, S'deir, Woschem und A'red bis zum Nedjran...“ Er fügt hinzu, ,[...] wenn ich nicht von meinem Erfolg fast hundertprozentig überzeugt wäre, würde ich nicht die Fortsetzung meiner Mission beantragen, da ich genau weiß, was man in Arabien riskiert, aber die Kenntnisse die ich von diesem Land habe und die guten Beziehungen die ich jetzt dort habe, ermöglichen es mir, noch mehr zu unternehmen. Außerdem wie ich es vorher erwähnt habe, was ich bis jetzt getan habe ist nur ein Teil und erst wenn alles gemacht ist, wird das Jetzige einen Wert haben"."

In einem Brief, immer noch aus Straßburg, vom 18. Mai $1882^{10}$, schreibt er, es scheine ihm, er habe überall alles besser beobachtet und mehr dabei gelernt als seine Vorgänger [...] Er wolle für seine nächste Reise keine Kopie mehr von den Inschriften machen, sondern nur noch jeweils einen Abklatsch davon ausführen, damit eine hundertprozentige Präcision gewährleistet werde ${ }^{11}$.

\section{Seine zweite und letzte Reise}

Die zweite Reise von Charles Huber in Arabien verlief von 1883 bis 1884 und war, wieder in einem Wort zusammengefaßt, ein Abenteuer voll großer Aufregungen und mit ständigem Unheil verbunden.

Am liebsten hätten wir Ihnen den letzten Brief von Charles Huber, den der Institut de France bis heute aufbewahrt hat, ungekürzt wiedergegeben. Aber da unsere Zeit bemessen ist, darf ich Ihnen nur einige übersetzte Auszüge davon vorlesen. Die wichtigsten Probleme dieser zweiten Reise sind darin, unserer Meinung nach, zusammengefasst.

So schreibt er aus Djeddah seinem elsässischen Freund, dem Dr. Weißgerber, am 6. Juli 1884 (er wird am 29. Juli ermordet) ${ }^{12}$,

a. Was Julius Euting betrifft: „Mein lieber Freund... Du wirst demnach mit Zufriedenheit hören, daß es Euting gelungen ist, die Stadt Hâil zu erreichen, aber daß ich ihn, dort angekommen, mit Hilfe des Emirs in Richtung Westen abschieben konnte. Ich nehme an, er ist dann in El Wegg am Roten Meer auf ein Schiff gegangen. Ich wußte, daß er die Absicht hatte, nach Djeddah zu kommen, wohin er seine Post übersenden ließ, aber nach meiner Ankunft habe ich erfahren, daß er hier nicht erschienen ist. Ich konnte ihn nicht hindern, in Hâil einzutreffen, genausowenig wie ich jeden $\mathrm{x}$ beliebigen daran hindern könnte! Kannst Du dies bitte gelegentlich den Berechtigten weitersagen. Aber einmal angekommen, verblieb mir nur noch, ihn weiterzuschicken und das war nicht leicht zustandezubringen, da er kein französischer Beauftragter war und über ganz andere Mittel verfügte als ich selbst...“

Bereits in seinen früheren Briefen wird Julius Euting von Charles Huber wie folgt erwähnt: „Professor Euting ist ein großer Gelehrter, einer der Männer der die semitischen Sprachen am besten kennt, er ist jedoch genauso enthusiastisch wie er gelehrt ist, und bevor er mir damals vorgeschlagen hatte, mit mir nach Arabien reisen zu können, ein Plan den er schon lange hegte, der aber viel stärker wurde als er von meiner Inschriftenentdeckung in Hedjaz hörte, hatte er bereits Urbi et Orbi 
bekanntgegeben, daß er mit mir gehen würde [...]“ - weiter im selben Text - „[...] aber es wird mit mir keinen Herrn Euting geben, was ich behaupte ist, daß ich mit ihm nicht mehr Beziehungen habe als mit anderen Personen, daß wir nicht zusammen reisen und daß, demzufolge, weder von einer Reisegemeinschaft noch von einer Unterordnung die Frage sein kann."

Es geht aus allem bereits 1883 klar hervor, daß Charles Huber von Anfang an auf seinen Reisen allein sein wollte, und das, ohne jegliche Hinterlist oder Falschheit ${ }^{13}$ wie zehn Jahre später unter Eutings Feder zulesen ist ${ }^{14}$.

b. Was die begrenzten Mittel von Charles Huber betrifft gibt es zwischen beiden Männern einen großen Unterschied. Julius Euting verfügt über bedeutende mehr oder weniger private Geldmittel, sowohl aus Württemberg wie auch aus Elsaß-Lothringen, wohingegen Charles Huber nur sehr beschränkte Mittel besitzt und zudem die von ihm jeweils erwarteten Kisten nie rechtzeitig eingetroffen sind.

Er schreibt à propos „Unterstützung“ weiter: „[...] Was willst Du (immer sein Freund, Dr. Weißgerber), es ist stärker als ich, ich kann mich nicht dazu bringen, betteln zu müssen. So habe ich lieber noch einmal einen Wechsel auf meine Mutter gezogen.... Ich hoffe, daß dieses Geld mir ermöglichen wird, über S'dous bis nach Baghdad zu kommen."

c. Über die Inschriften: Man kann es ihm nicht nachtragen, den wirklichen Wert der von ihm entdeckten Inschriften nicht sofort erkannt zu haben. Er war ja Erdkundler und kein Epigraphiker und hat Herrn Renan und Herrn Halevy seine Kopien und jeden Abklatsch immer sorgfältig zugesandt.

Was die Stele von Teima betrifft, so schreibt er noch eimal an Dr Weißgerber: „[...] Sie ist leider ein bißchen am oberen Drittel beschädigt, wenn dies nicht der Fall wäre, würde sie den bedeutendsten (phönizischen)[!] Text, den wir bis jetzt kennen, darstellen [...] Zu sagen welche Mühe wir hatten, diesen mächtigen Stein von Teima nach Hail zu transportieren und erst die Mühe die wir haben werden, um ihn dann auf Kamelrücken bis nach Baghdad zu bringen. Aber ich werde weder die Mühe noch die Schwierigkeiten scheuen, denn es freut mich viel zu sehr, diesen schönen Stein an Frankreich zu übergeben...“

26 Seine Freude und Begeisterung sind so groß, daß er auch in Teima mit Ausgrabungen anfangen möchte [...]: „Wenn ich reich wäre [...] ich liebe Arabien und die Wissenschaft arg genug, um diese Ausgrabungen auf meine Kosten zu unternehmen, [...] aber ich bin es nicht....". Weiter: „[...] sofern ich dort (d. h. in Baghdad) ankomme und kein Geld mehr in der Tasche habe, dann rufst Du (Dr Weißgerber ist natürlich hier gemeint) eine Spendenaktion im Elsaß zugunsten meiner Rückkehr auf. Ich weiß jetzt schon, daß sie erfolgreich sein wird.“

27 Und da spüren wir wieder, was wir rührend finden, welch starke Bande ihn mit seinem Elsaß verbinden. Er schreibt weiter, nachdem er sein Tagebuch und verschiedene Papiere und Berichte heimgeschickt hatte „Ich war gezwungen, diese Entscheidung zu treffen, denn, obwohl ich nichts davon erzähle, habe ich bei meiner letzten Reise durch das Gebiet der Ateibeh Araber zehn mal am Tag mein Leben aufs Spiel gesetzt. Somit wird wenigstens, sollte man mich umbringen, nicht alles verloren sein“".

28 Charles Huber ist sich der Gefahren sehr bewußt. In einem anderen Brief vom 3. Juli $1884^{15}$, immer noch aus Djeddah, schreibt er: „[...] ich verliere den Mut nicht und hoffe, daß das Glück das mir bis jetzt beigestanden ist, mich weiter begleiten wird [...] - weiter 
im Text seines letzten Briefes ${ }^{16}$ : - [...] wenn ich hier wenigstens einmal ein aufmunterndes Wort vom Ministerium oder von Herrn Maunoir erhalten hätte, wäre ich nach Ryadh gegangen, aber mein Leben umsonst riskieren, ohne ein einziges gutes Wort, da überlaß ich die Ehre den Anderen." Diese tiefe Verzweiflung, diese plötzliche Hoffnungslosigkeit, die schon in seinem Brief aus Damaskus vom 24. September $1883^{17}$ spürbar ist, wird hier deutlich.

Seine Liebe zu Arabien war jedoch stärker, denn einige Tage darauf, d.h. am 29. Juli 1884 , ist er dort ermordet worden.

Es ist offenkundig, daß diese wenigen kurzen Briefauszüge Charles Hubers arabische Pläne nur schwach und flüchtig widerspiegeln und daß das genauere Studium der noch nicht veröffentlichten französischen Briefdokumente es erlauben würde, die Arabienreisen dieser beiden Abenteurer in ihrem tatsächlichen Verlauf darzustellen.

PS : Cette brève présentation était, à l'évidence, proprement destinée à être lue durant le symposium de 1999 (cf. n. 1). Elle voulait, à partir des archives disponibles, insister sur le fait que le savant Julius Euting avait été largement précédé dans son exploration de l'Arabie par le géographe français Charles Huber et que celui-ci avait d'emblée conçu l'extrême importance de la stèle araméenne de Teima. Celle-ci, en effet, à cette époque, selon Ernest Renan, directeur du Collège de France, « mérite la seconde place parmi les monuments d'épigraphie orientale connus jusqu'ici » (CRAI, 1885, p. 107). Elle fut transportée, le 30 juin 1885, au Louvre (AO 1505) par le vice-concul de France à Djeddah, Jacques Félix de Lostalot-Bachoué ».

\section{NOTES}

1. Dieser Vortrag wurde beim internationalen Symposium „Julius Euting (1839-1913) und die Erforschung Arabiens" gehalten, das vom 11. bis zum 14. Juli 1999 in der Eberhard-KarlsUniversität in Tübingen stattgefunden hat. Die deutsche Fassung des Textes, die die Organisatoren der Tagung jahrelang versprochen hatten, in den Akten des Tübinger Symposiums zu veröffentlichen, wird jetzt von der Zeitschrift Anabases verlegt. Wir bedanken uns sehr bei Anabases. Die Vorträge des Symposiums wurden in Frankreich von S. Farès-Drappeau, Topoi 9/1 (1999), S. 479-481, und von H. Lozachmeur, Semitica 49 (1999), S. 197-199 kurz dargestellt.

Cette communication a été rédigée et prononcée en allemand durant le Symposium international «Julius Euting (1839-1913) und die Erforschung Arabiens », organisé du 11 au 14 juillet 1999, par l'Université Eberhard-Karl de Tübingen. Le texte de celle-ci, confié aux organisateurs du symposium en vue de sa publication dans les Actes de la rencontre, toujours promise pendant dix ans, trouve finalement son édition ici. Nous remercions Anabases pour l'accueil qu'il lui a réservé. Le symposium avait été brièvement présenté en France par S. Farès-Drappeau, dans Topoi 9/1 (1999), p. 479-481, et par H. Lozachmeur, dans Semitica 49 (1999), p. 197-199.

2. Diese Dokumente stammen aus Charles Hubers Archiven. Sie befinden sich im Institut de France (Paris) und sind unter dem Ref. G6 aufbewahrt (CIS). Wir bedanken uns herzlich bei Herrn Professor Jean Leclant, Secrétaire perpétuel de l'Académie des Inscriptions et Belles-Lettres, dafür, daß wir diese Briefe teilweise veröffentlichen dürfen.

3. Institut de France, G6, Charles Huber, Corr. Nr. 9. 
4. « [...] Si, à part cela ma présence à Paris devait vous faciliter ce que vous voulez bien faire pour moi, vous n'aurez qu'à m'en aviser, je partirai aussitôt malgré que [sic] ma situation s'aggrave ici et que je n'obtienne plus que difficilement des permis de séjour et d'absence. »

5. Institut de France, G6, Charles Huber, Corr. Nr.10. «[...] Pour moi, j’ai été expulsé en 1878 comme membre de la ligue d'Alsace et tout le reste de notre famille est mal notée dans le même sens. - À mon retour de ma mission, je n'ai obtenu qu'à grand peine, malgré les sentiments conciliants du Gouverneur d'Alsace-Lorraine, l'autorisation de pouvoir séjourner à Strasbourg chez ma mère [...] Tous les lundis, je suis obligé de me présenter à la préfecture de Police. Pour chaque absence et retour de ville il me faut des déclarations et des autorisations. À mon dernier retour de Paris, on m'a fait subir un vrai interrogatoire, pour savoir ce que j'y avais fait. Si donc je veux rester ici, au moins jusqu'à mon prochain départ pour l'Orient, il faut que je file doux... ». 6. Charles Huber, « Voyage dans l'Arabie centrale, Hamâd, Sammar, Qaçim, Hedjâz ", Bulletin de la Société de Géographie, $7^{\mathrm{e}}$ sér., 6 (1885), p. 92-148.

7. Vgl. Comptes rendus des séances de la Société de Géographie et de la Commission centrale, 1883, p. 214 (Rapp. Henri Duveyrier).

8. Institut de France, G6, Charles Huber, Corr. Nr. 2. (S. 6-8). « [...] Voici ce que j'aurai [sic] voulu ajouter comme conclusion à mon rapport. - Personnellement, je ne suis pas satisfait du résultat de ma mission, surtout si je considère ce qui reste à faire et ce que je suis sûr de pouvoir faire en Arabie et que j'aurai [sic] fait si on m'avait encore accordé une petite subvention. À vous, Monsieur, je n'ai pas besoin de démontrer l'intérêt qu'il y aurait à reprendre et continuer mon itinéraire primitif, continuer d'A'neïzah' ma marche sur R'iâd en passant par S'deir, le Woschem et l'A'red et arriver au Nedjran... »

9. «[...] Si je n'avais pas la quasi certitude de réussir je ne demanderai [sic] pas la continuation de ma mission, car je sais ce qu'on risque en Arabie, mais la connaissance du pays et les relations que j'y possède maintenant me permettent d'être plus entreprenant. - En outre, ainsi que je l'ai dit plus haut, ce que j'ai fait n'est qu'une partie, et ce n'est qu'en faisant le tout qu'elle aura une valeur."

10. Institut de France, G6, Charles Huber, Corr. Nr. 3.

11. So lautet der Text des Briefes(S. 8-11): « [...] Dans les nos 14 \& 16 du Globus de cette année j’ai vu une notice de M. Doughty sur ses parcours entre Kheïber, Hâil et Bereïdah et constaté avec plaisir que mes observations ne feront pas doublure sur presqu'aucun point avec les siennes, j'ai partout mieux observé et plus appris [...] Si l'on m'accorde la suite de ma mission j'ai l'intention de repasser rapidement par toutes les localités vues précédemment et de les relever à nouveau astronomiquement avec des instruments plus perfectionnés. En outre j'estamperai cette fois-ci toutes les inscriptions au lieu de les copier [...]. "

12. Institut de France, G6, Charles Huber, Corr. Nr 24. Dies ist der letzte Brief von Ch. Huber, den der Institut behält: « [... À propos de J. Euting] Tu apprendras donc avec satisfaction qu'il a pu arriver jusqu'à Hâil, mais qu'une fois là j'ai obtenu de l'émir de le faire filer par l'Ouest. Il a dû s'embarquer à El-Wegg sur la mer Rouge. Je savais qu'il avait l'intention de toucher Geddah où il s'était fait adresser sa correspondance, mais à mon arrivée ici, j'ai appris qu'il n'y avait pas paru. Je n'ai pas pu l'empêcher d'arriver à Hâil, pas plus que je ne pourrais en empêcher Pierre ou Paul, je te prie de dire cela à l'occasion à qui de droit, mais une fois là-bas je l'ai fait repartir, c'était tout ce que je pouvais faire. Et ce n'était pas facile à faire, car n'étant pas missionnaire français, il avait d'autres moyens que moi pour voyager [...]. »

13. Institut de France, G6. Siehe den Brief von Ernest Renan an Monsieur Euting aus Rosmapamon, am 8. Juli 1885.

14. Die Fotokopie dieses Memorandums (Straßburg, 17 novembre 1886) wurde uns dankenswerterweise von Dr Andreas Reichert zur Verfügung gestellt, woraus man dies lesen könnte.

15. Institut de France, G6. Charles Huber, Corr. Nr 23. 
16. Institut de France, G6. Vgl. nochmals zum Teil Corr. Nr 24.

17. Institut de France, G6. Vgl. Corr. Nr 20.

\section{AUTEURS}

\section{HÉLÈNE LOZACHMEUR}

Cabinet du Corpus Inscriptionum Semiticarum

Académie des Inscriptions et Belles-Lettres

\section{FRANÇOISE BRIQUEL-CHATONNET}

UMR 8167 Orient et Méditerranée - Mondes sémitiques

CNRS

francoise.briquel-chatonnet@ivry.cnrs.fr 